\title{
Recent results on spectra and yields from NA49
}

\author{
M. van Leeuwen for the NA49 collaboration*
}

S.V. Afanasiev ${ }^{9}$,T. Anticic ${ }^{21}$, B. Baatar ${ }^{9}$, D. Barna ${ }^{5}$, J. Bartke ${ }^{7}$, R.A. Barton ${ }^{3}$, M. Behler ${ }^{15}$, L. Betev ${ }^{10}$, H. Białkowska ${ }^{19}$, A. Billmeier ${ }^{10}$, C. Blume ${ }^{8}$, C.O. Blyth ${ }^{3}$, B. Boimska ${ }^{19}$, M. Botje ${ }^{1}$, J. Bracinik ${ }^{4}$, R. Bramm ${ }^{10}$, R. Brun ${ }^{11}$, P. Bunčić ${ }^{10,11}$, V. Cerny ${ }^{4}$, O. Chvala ${ }^{17}$, J.G. Cramer ${ }^{18}$, P. Csató ${ }^{5}$, P. Dinkelaker ${ }^{10}$, V. Eckardt ${ }^{16}$, P. Filip ${ }^{16}$, H.G. Fischer ${ }^{11}$, Z. Fodor ${ }^{5}$, P. Foka ${ }^{8}$, P. Freund ${ }^{16}$, V. Friese ${ }^{8,15}$, J. Gál ${ }^{5}$, M. Gaździcki ${ }^{10}$, G. Georgopoulos ${ }^{2}$, E. Gładysz ${ }^{7}$, S. Hegyi ${ }^{5}$, C. Höhne ${ }^{15}$, G. Igo ${ }^{14}$, P.G. Jones ${ }^{3}$, K. Kadija ${ }^{11,21}$, A. Karev ${ }^{16}$, V.I. Kolesnikov ${ }^{9}$, T. Kollegger ${ }^{10}$, M. Kowalski ${ }^{7}$ I. Kraus $^{8}$, M. Kreps ${ }^{4}$, M. van Leeuwen ${ }^{1}$, P. Lévai ${ }^{5}$, A.I. Malakhov ${ }^{9}$, S. Margetis ${ }^{13}$, C. Markert ${ }^{8}$, B.W. Mayes ${ }^{12}$, G.L. Melkumov ${ }^{9}$, C. Meurer $^{10}$, A. Mischke ${ }^{8}$, M. Mitrovski ${ }^{10}$, J. Molnár ${ }^{5}$, J.M. Nelson ${ }^{3}$, G. Pálla ${ }^{5}$, A.D. Panagiotou ${ }^{2}$, K. Perl ${ }^{20}$, A. Petridis ${ }^{2}$, M. Pikna ${ }^{4}$, L. Pinsky ${ }^{12}$, F. Pühlhofer ${ }^{15}$, J.G. Reid ${ }^{18}$, R. Renfordt ${ }^{10}$, W. Retyk ${ }^{20}$, C. Roland ${ }^{6}$, G. Roland ${ }^{6}$, A. Rybicki ${ }^{7}$, T. Sammer ${ }^{16}$, A. Sandoval ${ }^{8}$, H. Sann ${ }^{8}$, N. Schmitz ${ }^{16}$, P. Seyboth ${ }^{16}$, F. Siklér ${ }^{5}$, B. Sitar ${ }^{4}$, E. Skrzypczak ${ }^{20}$, G.T.A. Squier ${ }^{3}$, R. Stock ${ }^{10}$, H. Ströbele ${ }^{10}$, T. Susa ${ }^{21}$, I. Szentpétery ${ }^{5}$, J. Sziklai ${ }^{5}$, T.A. Trainor ${ }^{18}$, D. Varga ${ }^{5}$, M. Vassiliou ${ }^{2}$, G.I. Veres ${ }^{5}$, G. Vesztergombi ${ }^{5}$, D. Vranić ${ }^{8}$, S. Wenig ${ }^{11}$, A. Wetzler ${ }^{10}$, C. Whitten ${ }^{14}$, I.K. Yoo ${ }^{8,15}$, J. Zaranek ${ }^{10}$, J. Zimányi ${ }^{5}$

${ }^{1}$ NIKHEF, Amsterdam, Netherlands.

${ }^{2}$ Department of Physics, University of Athens, Athens, Greece.

${ }^{3}$ Birmingham University, Birmingham, England.

${ }^{4}$ Comenius University, Bratislava, Slovakia.

${ }^{5}$ KFKI Research Institute for Particle and Nuclear Physics, Budapest, Hungary.

${ }^{6}$ MIT, Cambridge, USA.

${ }^{7}$ Institute of Nuclear Physics, Cracow, Poland.

${ }^{8}$ Gesellschaft für Schwerionenforschung (GSI), Darmstadt, Germany.

${ }^{9}$ Joint Institute for Nuclear Research, Dubna, Russia.

${ }^{10}$ Fachbereich Physik der Universität, Frankfurt, Germany.

${ }^{11}$ CERN, Geneva, Switzerland.

${ }^{12}$ University of Houston, Houston, TX, USA.

${ }^{13}$ Kent State University, Kent, OH, USA.

${ }^{14}$ University of California at Los Angeles, Los Angeles, USA.

${ }^{15}$ Fachbereich Physik der Universität, Marburg, Germany.

${ }^{16}$ Max-Planck-Institut für Physik, Munich, Germany.

${ }^{17}$ Institute of Particle and Nuclear Physics, Charles University, Prague, Czech Republic.

${ }^{18}$ Nuclear Physics Laboratory, University of Washington, Seattle, WA, USA.

${ }^{19}$ Institute for Nuclear Studies, Warsaw, Poland.

${ }^{20}$ Institute for Experimental Physics, University of Warsaw, Warsaw, Poland.

${ }^{21}$ Rudjer Boskovic Institute, Zagreb, Croatia.

The energy dependence of hadron production in central $\mathrm{Pb}+\mathrm{Pb}$ collisions is presented

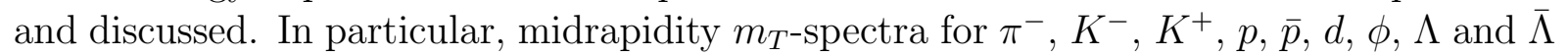
at 40, 80 and $158 \mathrm{AGeV}$ are shown. In addition $\Xi$ and $\Omega$ spectra are available at $158 \mathrm{AGeV}$. The spectra allow to determine the thermal freeze-out temperature $T$ and the transverse flow velocity $\beta_{T}$ at the three energies. We do not observe a significant energy dependence

\footnotetext{
*Presented at Quark Matter 2002, Nantes, France.
} 
of these parameters; furthermore there is no indication of early thermal freeze-out of $\Xi$ and $\Omega$ at $158 A \mathrm{GeV}$. Rapidity spectra for $\pi^{-}, K^{-}, K^{+}$and $\phi$ at 40,80 and $158 A \mathrm{GeV}$ are shown, as well as first results on $\Omega$ rapidity distributions at $158 \mathrm{AGeV}$. The chemical freeze-out parameters $T$ and $\mu_{B}$ at the three energies are determined from the total yields. The parameters are close to the expected phase boundary in the SPS energy range and above. Using the total yields of kaons and lambdas, the energy dependence of the strangeness to pion ratio is discussed. A maximum in this ratio is found at $40 A \mathrm{GeV}$. This maximum could indicate the formation of deconfined matter at energies above $40 A \mathrm{GeV}$. A search for open charm in a large sample of $158 \mathrm{AGeV}$ events is presented. No signal is observed. This result is compared to several model predictions.

\section{Introduction}

In the last few years NA49 has taken data at 40, 80 and 158 AGeV beam energy to study the energy dependence of particle production in nucleus-nucleus collisions and to search for indications of the onset of deconfinement in these collisions. In this paper recent results on particle spectra and yields are reported. Results by NA49 on other observables such as HBT, fluctuations and system size dependences from $\mathrm{p}+\mathrm{p}, \mathrm{p}+\mathrm{A}$, and $\mathrm{A}+\mathrm{A}$ (peripheral $\mathrm{Pb}+\mathrm{Pb}$, central $\mathrm{Si}+\mathrm{Si}$ and $\mathrm{C}+\mathrm{C}$ ) are presented elsewhere in these proceedings [1234].

In addition to the energy scan programme, a large dataset of central $\mathrm{Pb}+\mathrm{Pb}$ data has been collected at $158 \mathrm{AGeV}$, allowing to study rare particles such as $\Omega, D$, and $\phi \rightarrow \mathrm{e}^{+} \mathrm{e}^{-}$. Results on $\Omega$ and open charm production are presented here.

\section{Experiment and centrality selection}

The NA49 experimental setup [5] consists of four TPCs, two of which are in a magnetic field. The Main TPCs (MTPC) are outside the magnetic field and are used to identify particles by measuring the energy loss $d E / d x$. The particle identification capabilities of the MTPCs are augmented by two time-of-flight (TOF) detector arrays which have acceptance around midrapidity for kaons and close to midrapidity for protons and deuterons. Centrality selection of the events is done using a calorimeter which detects the projectile spectators. At 40 and $80 \mathrm{AGeV}$ the $7.2 \%$ most central events were selected. For the $158 \mathrm{AGeV}$ data the on-line centrality cut is at $10 \%$, but for the analysis of pions, kaons and $\phi$ an off-line cut was made at $5 \%$. The high statistics data sample used for the $\Omega$ analysis at $158 \mathrm{AGeV}$ was taken with a centrality trigger at $20 \%$ of the inelastic cross section.

\section{Transverse mass spectra and thermal freeze-out}

In Fig. 1 all available results on $m_{T}$-spectra as measured in central $\mathrm{Pb}+\mathrm{Pb}$ collisions by NA49 are collected. ${ }^{2}$ The spectra are measured at or close to midrapidity except for the $\phi, \Xi$ and $\Omega$ which are integrated over approximately 1 unit of rapidity, to increase statistics.

\footnotetext{
${ }^{2}$ The $\bar{p}, \Omega^{-}$and $\bar{\Omega}^{+} m_{T}$-spectra at $158 \mathrm{AGeV}$ appearing in the printed version of these proceedings (Nucl. Phys. A715 161c) are unfortunately incorrect and therefore differ slightly from those presented here.
} 


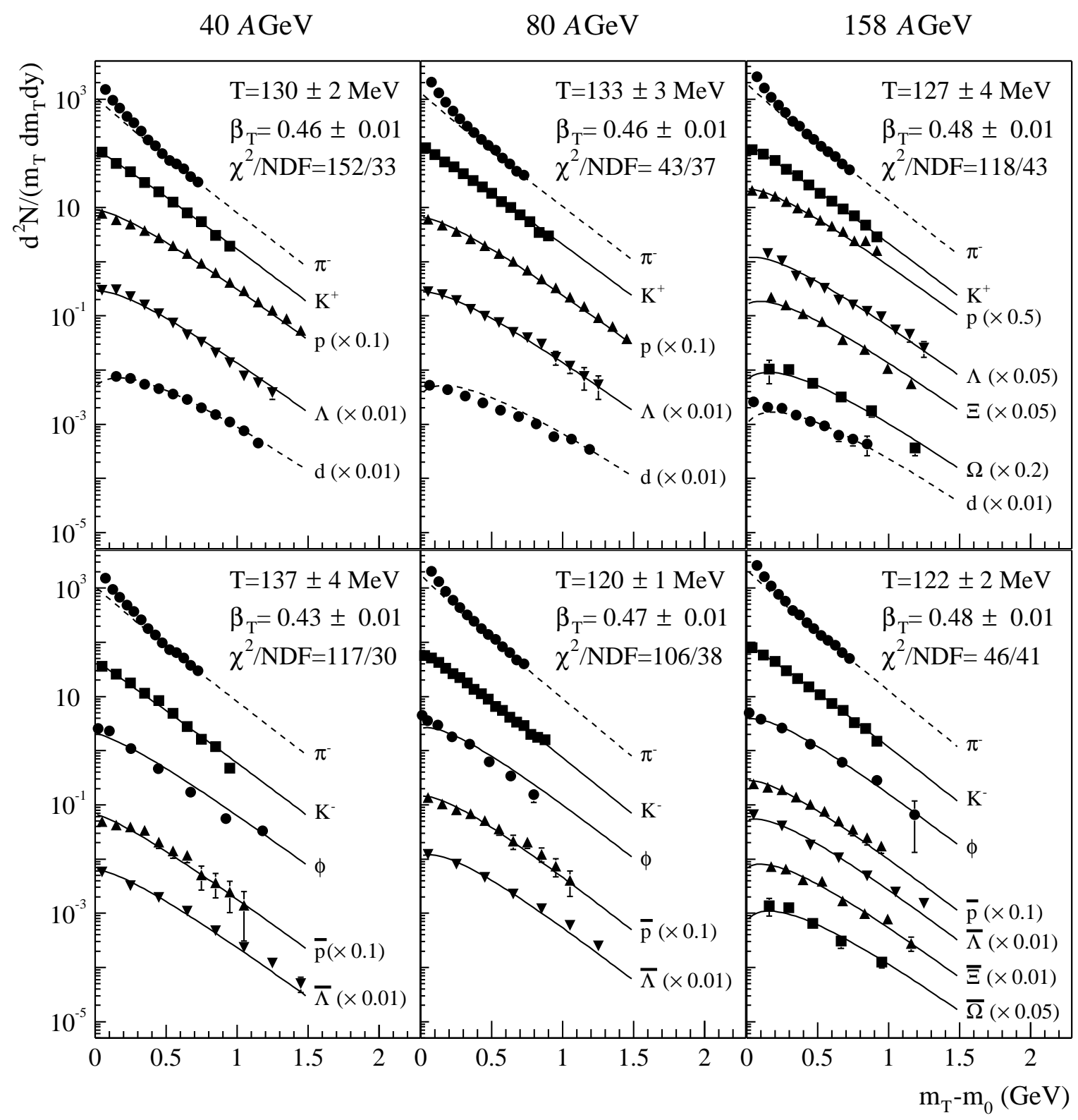

Figure 1. Transverse mass spectra in central 40 (left), 80 (middle) and $158 A \mathrm{GeV}$ (right) $\mathrm{Pb}+\mathrm{Pb}$ collisions. The lines show the result of the transverse flow fit as described in the text. 
The $\Xi$ and $\phi$ at $158 A \mathrm{GeV}$ have already been published [67] while the kaon and pion spectra are submitted for publication [9. All other presented results are still preliminary.

In a hydro-dynamical picture, the $m_{T}$-spectra of particles are sensitive to transverse flow. To characterise the flow, the spectra were fitted with $[8$

$\frac{d N}{m_{T} d m_{T} d y} \propto m_{T} K_{1}\left(\frac{m_{T} \cosh \rho}{T}\right) I_{0}\left(\frac{p_{T} \sinh \rho}{T}\right)$.

A combined fit of several spectra with this function allows to determine the thermal freezeout temperature $T$ and the mean transverse flow velocity $\beta_{T}\left(\rho=\operatorname{atanh} \beta_{T}\right)$. The $\pi^{-}$ and deuteron spectra were excluded from the fit (dashed lines in Fig. 1) because the pions are expected to have a significant contribution from resonance decays and the deuterons may be formed by coalescence. The particles (baryons and $K^{+}$) and anti-particles (antibaryons and $K^{-}$) were fitted separately. The $\phi$ was included in the anti-particle fit, because it is not expected to be sensitive to the baryon density. The fit describes the data reasonably well, even including the pions at higher $m_{T}$ and the deuterons. The relatively large $\chi^{2}$ per degree of freedom can partly be explained by the fact that only statistical errors were taken into account in the fit (systematic errors are not yet available for all spectra). Since the slopes of the $m_{T}$-spectra of particles and anti-particles are similar, the differences between the freeze-out parameters for both groups of particles are small.

The differences between the three different energies are not larger than the difference between the particles and anti-particles at each energy, implying that the energy dependence of the thermal freeze-out conditions is small at these energies.

The $\Xi$ and $\Omega$ spectra, which are only available at $158 A \mathrm{GeV}$, do not show large deviations from the fit. Thus, based on these data, there is no indication of an early freeze-out as has been suggested by several authors [1011]. The slope parameters of the $\Omega$ spectra are compatible with earlier measurements by WA97 [12].

\section{Rapidity spectra and baryon density}

The $m_{T}$-spectra of kaons as shown in Fig. 11 were obtained using combined $d E / d x$ and TOF information at midrapidity. The $d E / d x$ measurement in the TPC alone allows to identify kaons at forward rapidities. The resulting spectra are shown in Fig. 2, together with $\pi^{-}$distributions which were obtained using unidentified negatively charged particles, corrected for contributions of $K^{-}, \bar{p}$ and non-vertex tracks.

The $K^{-}$distributions are narrower than the $K^{+}$distributions at all energies. This could indicate that the kaons are sensitive to the local baryon density, which is reflected in the difference between the $\Lambda$ and $\bar{\Lambda}$ spectra as well (for details on the $\Lambda$ analysis, see [13] in these proceedings).

The $\phi$ spectra are obtained from invariant mass distributions of $K^{+} K^{-}$pairs. The resulting rapidity spectra are shown in Fig. 3, where the new results at 40 and $80 A \mathrm{GeV}$ are compared to the published result at $158 A \mathrm{GeV}$ [7. Clearly, the width of the distribution, as well as the total yield, increases with beam energy.

The large statistics data sample of the $20 \%$ most central $\mathrm{Pb}+\mathrm{Pb}$ collisions at $158 \mathrm{AGeV}$ beam energy, which was taken in the year 2000, has been used for the $\Omega$ analysis. The resulting $m_{T}$-spectra are shown in Fig. 1 and the corresponding rapidity spectra in Fig. 4 , 


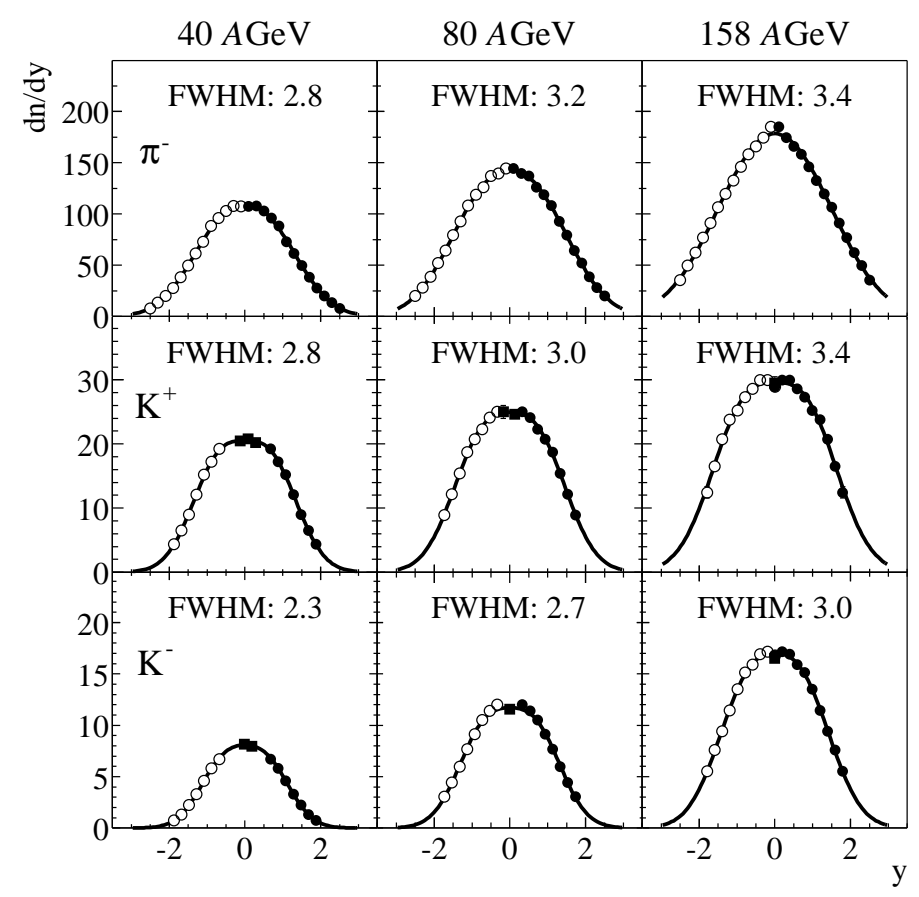

Figure 2. Rapidity spectra for kaons and pions at three different energies. The open points are reflected around midrapidity. For kaons, the circles indicate the result of the $d E / d x$ analysis, while the squares are the result of the combined $d E / d x$-TOF analysis. The distributions are fitted with a double Gaussian and the resulting full width at half maximum (FWHM) is indicated in the figure.

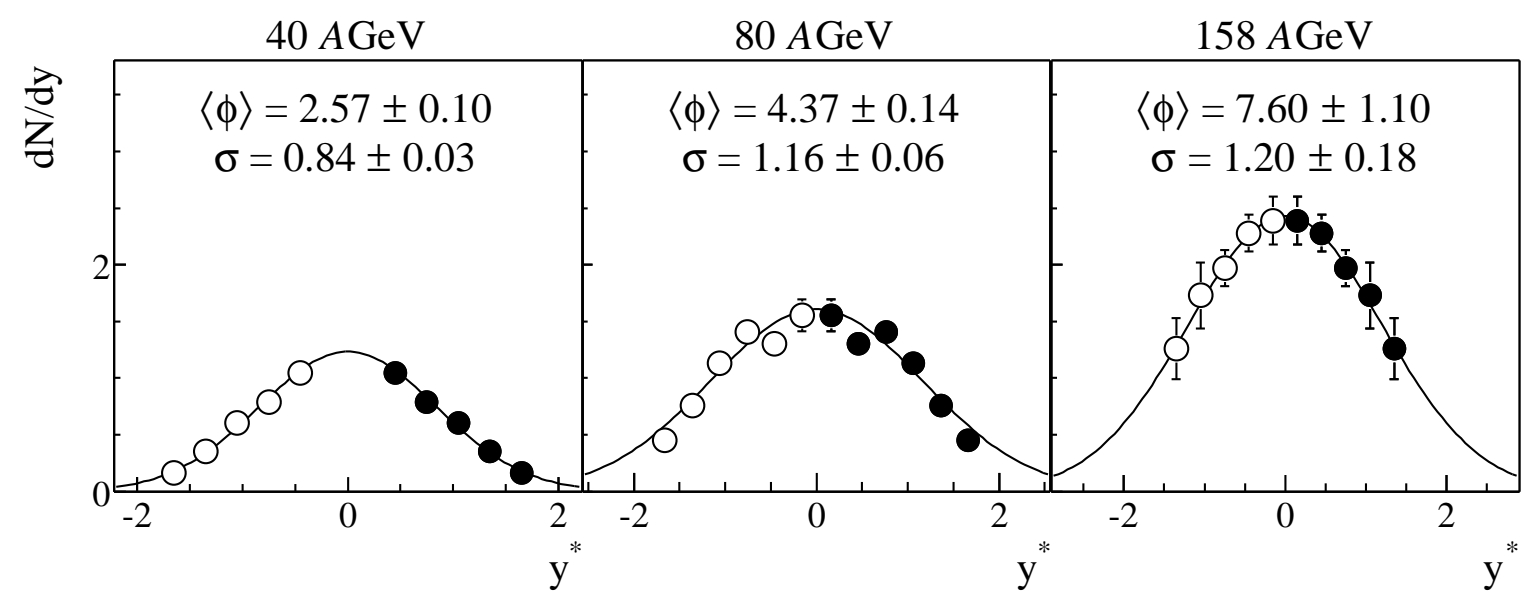

Figure 3. Rapidity spectra of $\phi$ production at 40,80 and $158 A \mathrm{GeV}$ respectively. The open points are reflected around mid-rapidity. The full lines show a Gaussian fit, used to obtain the mean multiplicities in full phase space $\langle\phi\rangle$. The fitted widths $\sigma$ are given as well. 

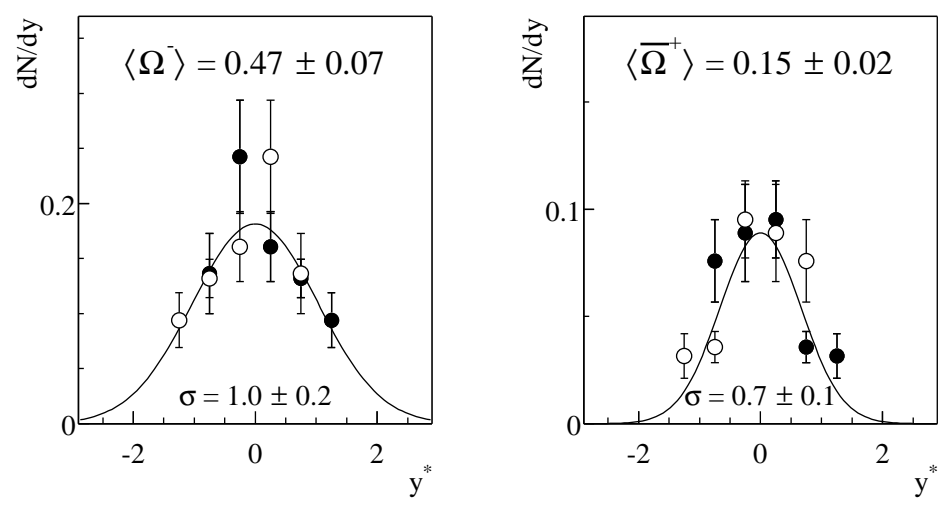

Figure 4. Rapidity spectra of $\Omega^{-}$and $\bar{\Omega}^{+}$at $158 A \mathrm{GeV}$. The total yields $\langle\Omega\rangle$ were calculated by integrating the fitted Gaussians (full line). The widths $\sigma$ of the fitted curves are also given.

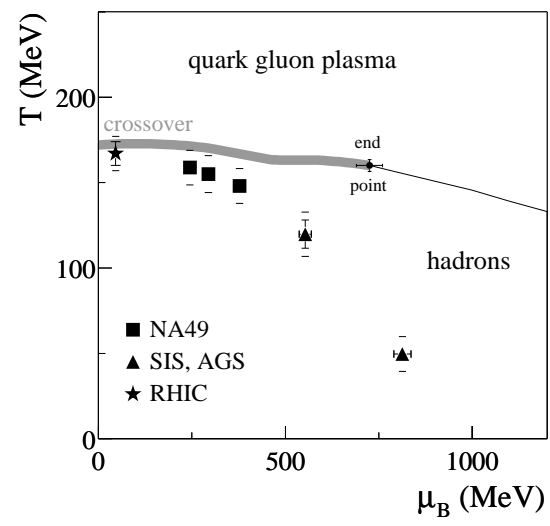

Figure 5. Chemical freeze-out points as determined from a hadron gas fit at different energies [15]. The crossover curve is taken from [16].

Integrating over rapidity gives mean multiplicities per event of $\left\langle\Omega^{-}\right\rangle=0.47 \pm 0.07$ and $\left\langle\bar{\Omega}^{+}\right\rangle=0.15 \pm 0.02$. The observation that the ratio $\bar{\Omega}^{+} / \Omega^{-}$is below unity cannot be explained in a pure string fragmentation model but fits to expectations in an equilibrium hadron gas model 14. Also note that the $\Omega^{-}$has a slightly wider rapidity distribution than the $\bar{\Omega}^{+}$. This indicates that even the $\Omega$ is sensitive to the effect of baryon density.

\section{Total yields and chemical freeze-out}

The measured rapidity spectra have been integrated to obtain the mean multiplicities in full phase space. To determine the chemical freeze-out parameters at the three different energies, the total yields were fitted with a hadron gas model with partial strangeness saturation [15]. The obtained temperatures for chemical freeze-out $(145-160 \mathrm{MeV})$ are larger than the thermal freeze-out temperatures (115-140 MeV). The chemical freeze-out temperature $T$ and baryon chemical potential $\mu_{B}$ are compared to results at AGS and RHIC in Fig. 5. The freeze-out points follow a smooth curve of increasing temperature and decreasing baryon density with energy. The observed freeze-out parameters are close to the calculated phase boundary [16] at SPS energies and above.

\section{Energy dependence of strangeness production}

The mean multiplicities of the most abundant carriers of strangeness, kaons and lambdas, are plotted as a function of the centre of mass energy in Fig. 6. Only the charged kaons are shown because the neutral kaons are not measured at all energies. All yields are divided by the total pion yield $\langle\pi\rangle=1.5\left(\left\langle\pi^{+}\right\rangle+\left\langle\pi^{-}\right\rangle\right)$. It is seen that the $\left\langle K^{+}\right\rangle /\langle\pi\rangle$ ratio peaks at $40 \mathrm{AGeV}$, whereas $\left\langle K^{-}\right\rangle /\langle\pi\rangle$ increases monotonically. On the other hand, 

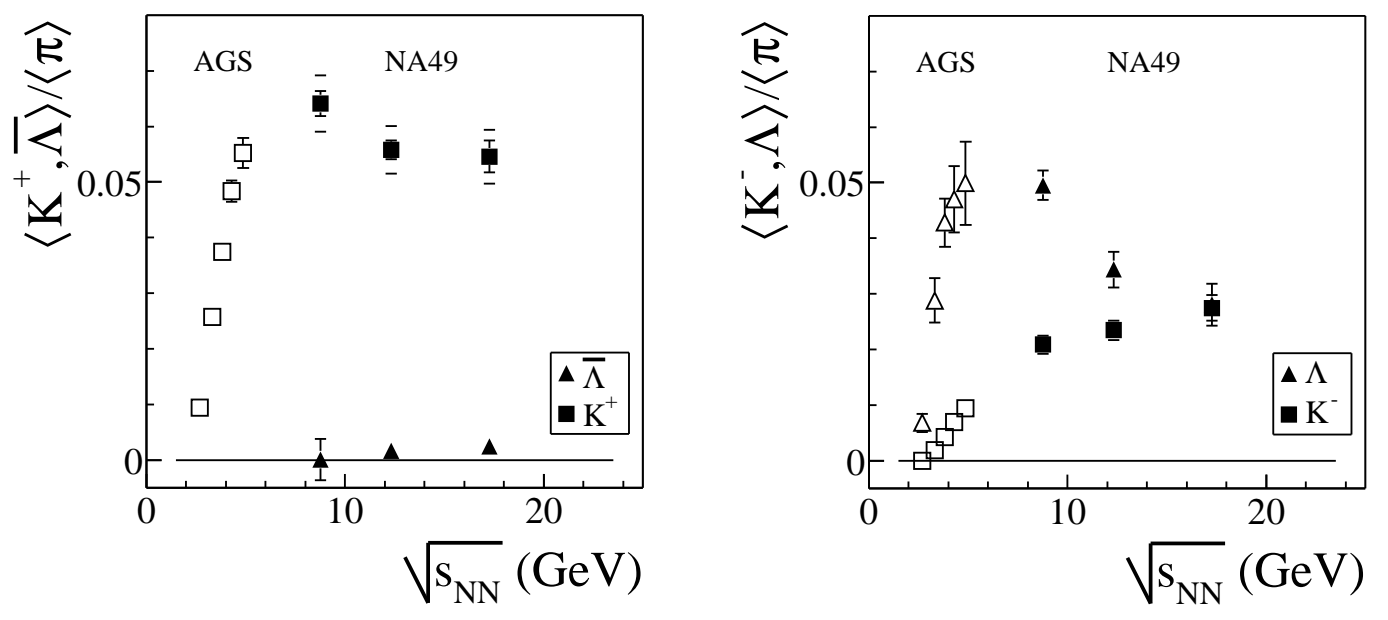

Figure 6. Total yields of $K^{+}$and $\bar{\Lambda}$ (left) and $K^{-}$and $\Lambda$ (right), normalised to the total pion multiplicity, as a function of $\sqrt{s}$ for central $\mathrm{Pb}+\mathrm{Pb}(\mathrm{NA} 49)$ and $\mathrm{Au}+\mathrm{Au}(\mathrm{AGS})$ collisions.
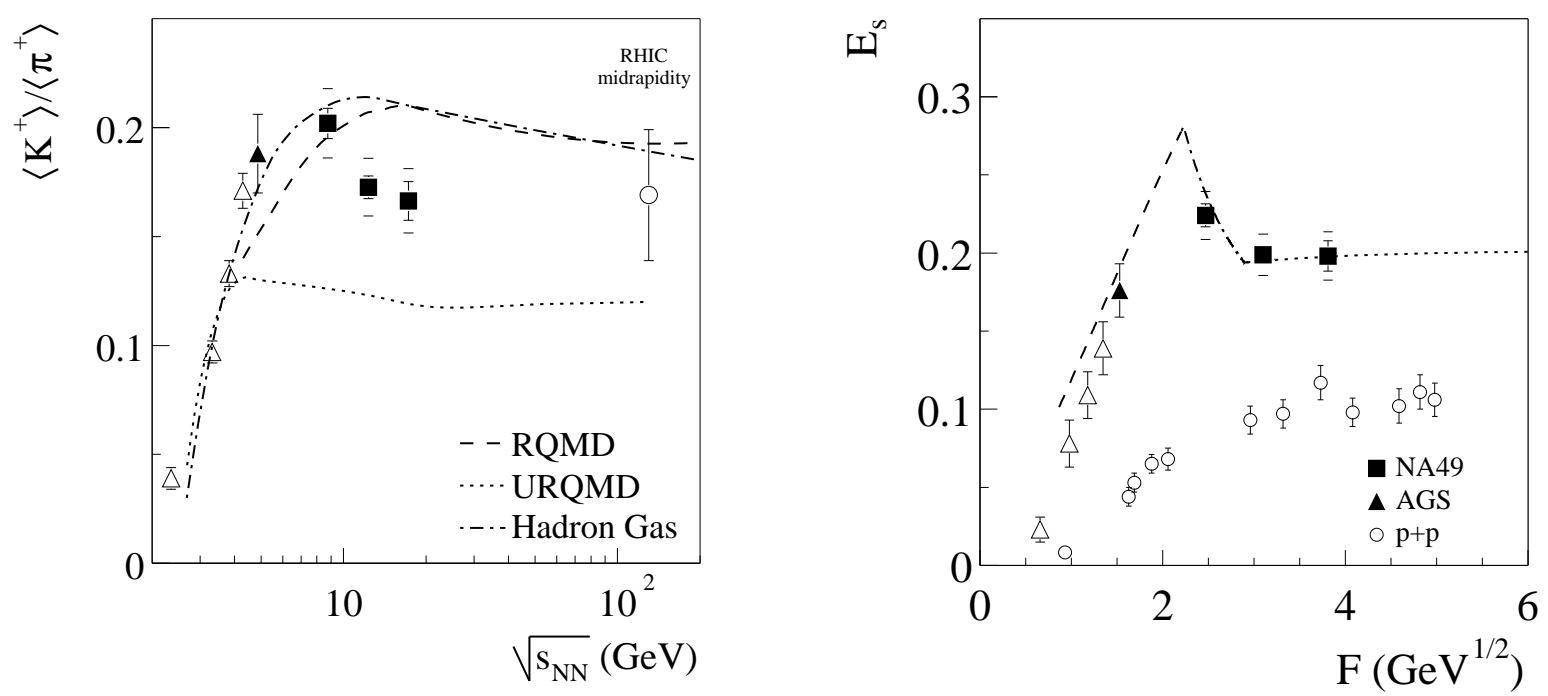

Figure 7. Energy dependence of $\left\langle K^{+}\right\rangle /\langle\pi\rangle$ compared to different model predictions which do not invoke QGP formation (see text).
Figure 8. Comparison of the strangeness to entropy ratio $E_{S}$ to a model calculation [20] which does assume that a QGP is formed above approximately $30 \mathrm{AGeV}$. 
the $\Lambda$ production also peaks around $40 A \mathrm{GeV}$, whereas the $\bar{\Lambda}$ yield increases monotonically with energy. Apparently, both the total $s$ production and the $\bar{s}$ production have a maximum close to $40 \mathrm{AGeV}$. The maximum in the $\bar{s}$ production is directly seen $K^{+} / \pi$ ratio because the contribution of $\bar{\Lambda}$ is small. Although the $K^{-}$represent a large fraction of the $s$ production, the maximum in the $s$ production is reflected in the $\Lambda / \pi$ ratio.

In Fig. [7 the energy dependence of the $\left\langle K^{+}\right\rangle /\left\langle\pi^{+}\right\rangle$ratio is compared to several models which do not assume the formation of a QGP in the collision. The UrQMD model [17] fails to describe the data because the pion yields are over-estimated by about $30 \%$. The RQMD model [18] gives a much better descriptions, but over-estimates the ratios at 80 and $158 \mathrm{AGeV}$. The same is true for the hadron gas model [19]. In this model, the $\left\langle K^{+}\right\rangle /\left\langle\pi^{+}\right\rangle$ratio peaks around $40 \mathrm{AGeV}$ due to the interplay of the decreasing baryon density and the increasing temperature with energy.

In Fig. 8] the energy dependence $\left(F \equiv\left(\sqrt{s}-2 m_{N}\right)^{3 / 4} / \sqrt{s}^{1 / 4}\right)$ of the strangeness to entropy ratio $E_{S}=(\langle\Lambda\rangle+\langle K\rangle+\langle\bar{K}\rangle) /\langle\pi\rangle$ is compared to a model [20] which assumes that above a certain energy a QGP is formed in the collision. The peak in the model curve is caused by this transition. In [9] it is shown that the model also describes the energy dependence of pion production.

NA49 intends to complete the energy scan by taking data at 20 and $30 \mathrm{AGeV}$ in 2002 .

\section{Open charm upper limit}

All available central $\mathrm{Pb}+\mathrm{Pb}$ data at $158 \mathrm{AGeV}$ (900k events at $10 \%$ and $3 \mathrm{M}$ events at $20 \%$ centrality) were used in an invariant mass analysis to measure open charm. The
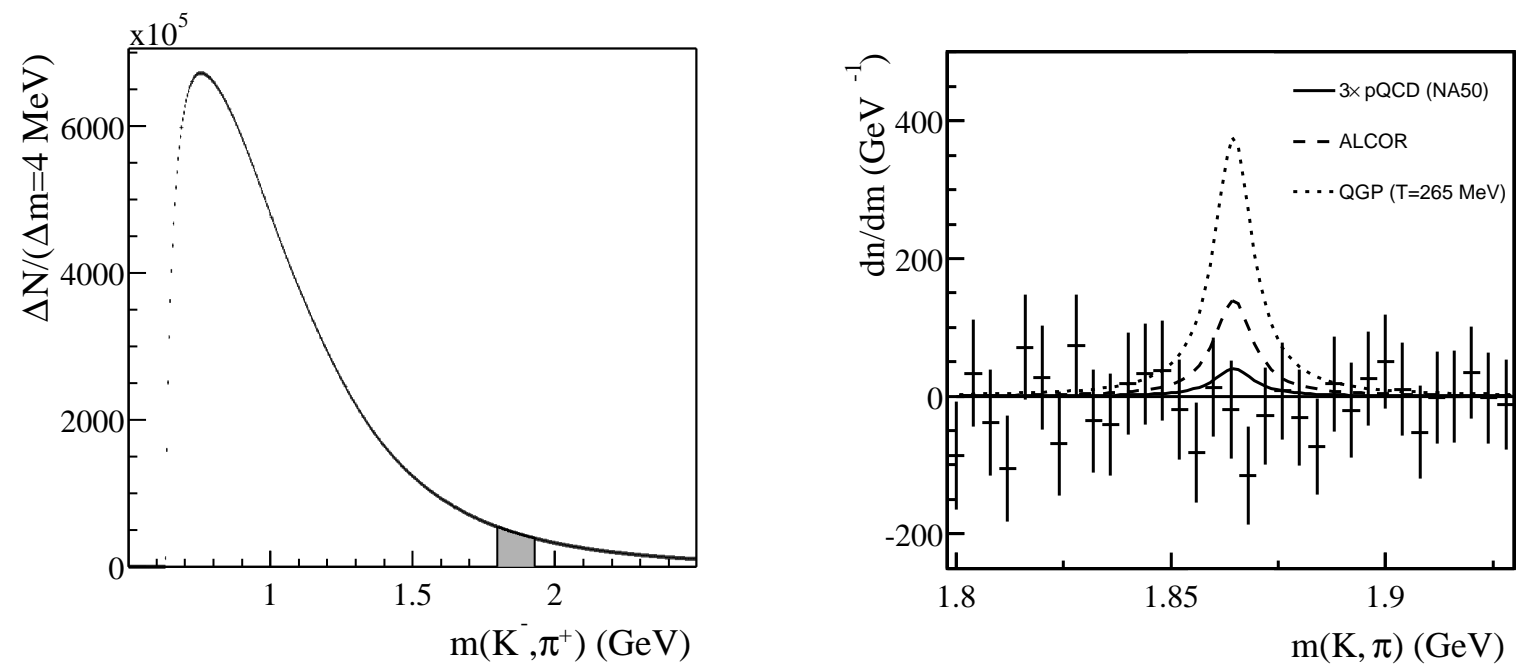

Figure 9. Uncorrected invariant mass distribution of all $K^{-}, \pi^{+}$pairs (left) and invariant mass distribution for the sum of all $D^{0}$ and $\bar{D}^{0}$ candidates after background subtraction (right), corrected for acceptance and efficiency. The curves indicate predictions of various models (see text). 
invariant mass was calculated for pairs of pion and kaon candidates, to detect the decays $D^{0} \rightarrow K^{-}+\pi^{+}$and $\bar{D}^{0} \rightarrow K^{+}+\pi^{-}$,

which have a branching fraction of 3.83\%. For tracks which reach the MTPC, the kaon candidates were selected by $d E / d x$ measured in the MTPC. For tracks which do not reach the MTPC, all candidates were used. The invariant mass histogram for $D^{0}$ before background subtraction is shown in the left panel of Fig. 9. The result after adding the $\bar{D}^{0}$, subtracting the background and correcting for acceptance and efficiency losses is shown in the right panel of Fig. 9. No signal of the decays is visible. Detector simulations have shown that the shape of the expected signal can be parametrised as a Breit-Wigner with a width $\Gamma=11 \mathrm{MeV}$. Using this parametrisation, predictions by different models are shown in the figure. It is seen that thermal production of charm quarks in a Quark Gluon Plasma at $T=265 \mathrm{MeV}$, as described in [21], is excluded (dotted curve in Fig. 9). The expectation from the quark coalescence model ALCOR [22] is at the limit of the sensitivity of the present analysis (dashed curve). Lower values of the charm yield, such as the expectation from perturbative QCD, or a factor 3 enhancement [23] (full curve) cannot be excluded. In order to quantify the result, the data have been fitted with the parametrised peak shape, leaving the normalisation as the only free parameter. The result is $\left\langle D^{0}+\bar{D}^{0}\right\rangle=-1.07 \pm 0.84$.

\section{Summary and outlook}

Spectra of $\pi, K, p, d, \phi$, and $\Lambda$ in central $\mathrm{Pb}+\mathrm{Pb}$ collisions at 40,80 and $158 A \mathrm{GeV}$ are presented, together with new results on $\Omega$ rapidity and $m_{T}$ spectra at $158 \mathrm{AGeV}$.

The $m_{T}$-spectra are compatible with radial flow, with similar temperature $T \approx 130 \mathrm{MeV}$ and transverse flow velocity $\beta_{T} \approx 0.45$ at the different energies. The $\Xi$ and $\Omega$ spectra, which are only measured at $158 A \mathrm{GeV}$, do not show indications of early freeze-out.

The chemical freeze-out parameters as determined from the measured total yields approach the phase boundary in the SPS energy range.

The energy dependence of strangeness production shows a maximum in the relative strangeness yield at $40 \mathrm{AGeV}$. This maximum is observed in the $K^{+} / \pi$ ratio for the $\bar{s}$ and in the $\Lambda / \pi$ ratio for the $s$ quarks. The maximum can be explained by the onset of deconfinement in the energy range between AGS and SPS. Upcoming runs at 20 and $30 \mathrm{AGeV}$ will provide a more accurate localisation of the maximum.

To investigate open charm production in heavy ion collisions, an invariant mass analysis has been performed using a large sample of $158 A \mathrm{GeV} \mathrm{Pb}+\mathrm{Pb}$ events. No signal has been observed. The result excludes equilibrium charm production in a QGP of $T=265 \mathrm{MeV}$.

\section{Acknowledgements}

We would like to thank F. Becattini for providing the results of his hadron gas fits.

This work was supported by the Director, Office of Energy Research, Division of Nuclear Physics of the Office of High Energy and Nuclear Physics of the US Department of Energy (DE-ACO3-76SFOOO98 and DE-FG02-91ER40609), the US National Science Foundation, the Bundesministerium fur Bildung und Forschung, Germany, the Alexander von Humboldt Foundation, the UK Engineering and Physical Sciences Research Council, 
the Polish State Committee for Scientific Research (5 P03B 13023 and 2 P03B 02418), the Hungarian Scientific Research Foundation (T14920 and T32293), Hungarian National Science Foundation, OTKA, (F034707), the EC Marie Curie Foundation, and the PolishGerman Foundation.

\section{REFERENCES}

1. C. Blume for the NA 49 collaboration, these proceedings.

2. M. Kreps for the NA49 collaboration, these proceedings.

3. C. Höhne for the NA49 collaboration, these proceedings.

4. H.-G. Fischer, these proceedings.

5. S. V. Afanasiev et al. [NA49 Collaboration], Nucl. Instrum. Meth. A 430 (1999) 210.

6. S. V. Afanasiev et al. [NA49 Collaboration], Phys. Lett. B 538 (2002) 275 arXiv:hep-ex/0202037.

7. S. V. Afanasiev et al. [NA49 Collaboration], Phys. Lett. B 491 (2000) 59.

8. E. Schnedermann and U. W. Heinz, Phys. Rev. C 50 (1994) 1675 arXiv:nucl-th/9402018.

9. S. V. Afanasiev et al. [NA49 Collaboration], arXiv:nucl-ex/0205002, submitted for publication in Phys. Rev. C.

10. H. van Hecke, H. Sorge and N. Xu, Phys. Rev. Lett. 81 (1998) 5764 arXiv:nucl-th/9804035.

11. A. Dumitru, S. A. Bass, M. Bleicher, H. Stocker and W. Greiner, Phys. Lett. B 460 (1999) 411 arXiv:nucl-th/9901046. M. Bleicher, these proceedings.

12. F. Antinori et al. [WA97 Collaboration], Nucl. Phys. A 661 (1999) 481.

13. A. Mischke for the NA49 collaboration, these proceedings.

14. S. A. Bass et al., arXiv:nucl-th/0204049.

15. F. Becattini et al, manuscript in preparation.

F. Becattini, M. Gaździcki and J. Sollfrank, Eur. Phys. J. C 5 (1998) 143 arXiv:hep-ph/9710529.

16. Z. Fodor and S. D. Katz, JHEP 0203 (2002) 014 arXiv:hep-lat/0106002.

17. H. Weber, E. L. Bratkovskaya and H. Stocker, arXiv:nucl-th/0205030. S.A. Bass et al. [UrQMD collaboration], Prog. Part. Nucl. Phys. 41 (1998) 255

18. F. Wang, H. Liu, H. Sorge, N. Xu and J. Yang, Phys. Rev. C 61 (2000) 064904.

19. P. Braun-Munzinger, J. Cleymans, H. Oeschler and K. Redlich, Nucl. Phys. A 697 (2002) 902 arXiv:hep-ph/0106066.

20. M. Gaździcki and M. I. Gorenstein, Acta Phys. Polon. B 30 (1999) 2705 arXiv:hep-ph/9803462.

21. M. Gaździcki and C. Markert, Acta Phys. Polon. B 31 (2000) 965 arXiv:hep-ph/9904441.

22. P. Lévai, T. S. Biró, P. Csizmadia, T. Csörgö and J. Zimányi, J. Phys. G 27 (2001) 703 arXiv:nucl-th/0011023.

23. M. C. Abreu et al. [NA38 Collaboration], Eur. Phys. J. C 14 (2000) 443. 